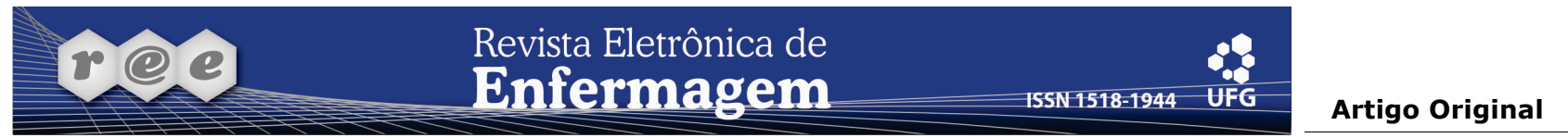

\title{
Processo de formação de contas em um hospital de ensino especializado em cardiologia e pneumonia
}

The billing process at a teaching hospital specialized in cardiology and pulmonology

Proceso de formación de cuentas en un hospital de enseñanza especializado en cardiología y neumología

\section{Gabriela Favaro Faria Guerrer ${ }^{1}$, Valéria Castilho ${ }^{2}$, Antônio Fernandes Costa Lima ${ }^{3}$}

\footnotetext{
${ }^{1}$ Enfermeira, Mestre em Enfermagem. São Paulo, SP, Brasil. E-mail: gabriela.faria@incor.usp.br.

${ }^{2}$ Enfermeira, Doutora em Enfermagem. Professora Associada da Escola de Enfermagem da Universidade de São Paulo (EE/USP). São Paulo, SP, Brasil. E-mail: valeriac@usp.br.

${ }^{3}$ Enfermeiro, Doutor em Enfermagem. Professor Doutor da EE/USP. São Paulo, SP, Brasil. E-mail: tonifer@usp.br.
}

\section{RESUMO}

Esta pesquisa exploratória objetivou descrever e mapear o processo de formação de contas em um hospital público universitário, de nível terciário, especializado em cardiologia e pneumologia. No período de maio a junho de 2012 , procedeu-se à identificação e documentação das etapas do processo que foram validadas junto aos profissionais envolvidos na auditoria de contas hospitalares. Evidenciou-se que, no momento da pré-análise das contas, os auditores realizam correções para fundamentar a cobrança dos procedimentos e evitar glosas e perdas de faturamento. O mapeamento do processo permitiu a proposição de estratégias visando minimizar o tempo de apresentação de contas às fontes pagadoras. Ao conferir visibilidade à dinâmica deste processo, fundamental para o equilíbrio econômico-financeiro do hospital estudado, torna-se esse conhecimento de domínio público e acessível a outras organizações de saúde que queiram incrementar o seu faturamento e reduzir as divergências entre o prontuário clínico e a conta hospitalar do paciente.

Descritores: Documentação; Auditoria Clínica; Faturamento; Institutos de Cardiologia.

\section{ABSTRACT}

The objective of this exploratory study was to describe and map out the billing process in a public tertiary-level university hospital specialized in cardiology and pulmonology. In the period between May and June of 2012, we identified and documented the steps in the process validated by the professionals involved in the hospital bill audit service. We found that during billing pre-analysis, auditors make corrections to justify the billing of procedures and to avoid unwarranted billing and loss of revenue. Mapping out the process allowed us to propose strategies to minimize the time for presenting bills to payment sources. By bringing visibility to this process, which is fundamental for the economic-financial balance of the studied hospital, we bring such knowledge to the public domain. Thus, it is accessible to other health organizations that wish to increment their revenue and reduce divergences between patient charts and the patient's hospital bill.

Descriptors: Documentation; Clinical Audit; Billing; Cardiac Care Facilities.

\section{RESUMEN}

Se objetivó describir y mapear el proceso de formación de cuentas en un hospital público universitario terciario especializado en cardiología y neumología. Entre mayo y junio de 2012 se procedió a identificar la documentación de las etapas del proceso, validadas con los profesionales involucrados en la auditoría de cuentas hospitalarias. Se evidenció que antes del análisis de cuentas, los auditores realizan correcciones para fundamentar la cobranza de los procedimientos y evitar divergencias y pérdidas de facturación. El mapeo del proceso permitió la propuesta de estrategias orientadas a disminuir el tiempo de presentación de cuentas a las fuentes de pago. Al otorgársele visibilidad a la dinámica del proceso, fundamental para el equilibrio económico-financiero del hospital estudiado, ese conocimiento se vuelve de dominio público y es accesible para otras organizaciones de salud que quieran incrementar su facturación y reducir las divergencias entre las historias clínicas y la cuenta hospitalaria del paciente.

Descriptores: Documentación; Auditoría Clínica; Facturación; Instituciones Cardiológicas. 


\section{INTRODUÇÃO}

Grandes empresas têm se preocupado em utilizar a auditoria, de forma contínua, a fim de assegurar a qualidade dos serviços prestados aos clientes, que estão cada vez mais convictos de seus direitos ${ }^{(1)}$.

As organizações hospitalares constituem empresas de serviços complexas e podem, por meio da auditoria, melhorar os seus processos de trabalho, incrementar os recursos (humanos, estruturais e materiais) disponíveis e aumentar a eficácia dos resultados que pretendem alcançar.

$\mathrm{Na}$ área da saúde a auditoria surgiu com a finalidade de avaliar a qualidade da assistência prestada ao paciente, mas a competitividade, o avanço tecnológico e os tratamentos crescentemente dispendiosos despertaram nos profissionais que atuam na área, a necessidade de desenvolver a visão financeira contábil ${ }^{(2)}$.

Estudo de revisão sistemática da literatura, que analisou a produção científica nacional sobre auditoria em enfermagem, no período de 1998 a 2008, evidenciou a realização de pesquisas quantitativas do tipo descritivo-exploratório ou exploratório, de cunho documental e retrospectivo em hospitais/instituições de saúde vinculadas à iniciativa privada. Comprovou que a temática ainda é pouco explorada sendo necessários novos estudos que busquem além de quantificar os erros presentes nas anotações de enfermagem e os prejuízos decorrentes de tais inconformidades, conhecer, discutir e analisar as práticas desenvolvidas por enfermeiros com relação à auditoria e os aspectos contextuais intervenientes na qualidade dos registros de enfermagem ${ }^{(3)}$.

A auditoria possibilita a verificação da compatibilidade entre os procedimentos realizados e a cobrança dos itens componentes das contas hospitalares. Esses itens são estabelecidos, contratualmente, entre o prestador de serviços e as operadoras de planos de saúde (OPS) considerando os insumos utilizados no tratamento do paciente, tais como diárias, taxas administrativas, honorários médicos, serviços diagnósticos, materiais, medicamentos e gases ${ }^{(4)}$.

As contas hospitalares podem ser auditadas por intermédio da pré-auditoria ou auditoria prospectiva que consiste na avaliação dos procedimentos médicos antes de sua realização; da auditoria concorrente ou proativa que compreende a análise pericial ligada ao tratamento ou procedimento ao qual o paciente será submetido durante a sua internação, e da auditoria retrospectiva ou de revisão de contas que abarca a análise pericial dos procedimentos médicos realizados com ou sem apreciação do prontuário clínico ${ }^{(5)}$.

Um dos autores do presente estudo integra a equipe de auditora de contas de um hospital público universitário de alta complexidade - especializado em cardiologia, pneumologia e cirurgias cardíaca e torácica que se dedica à assistência, ao ensino, à pesquisa e à extensão universitária à sociedade ${ }^{(6)}$.

O Hospital em questão possui 500 leitos distribuídos em sete unidades de internação e seis Unidades de Terapia Intensiva de alta complexidade. O Centro Cirúrgico, com 14 salas de operação e produção média de 20 cirurgias por dia, é um dos mais modernos da América Latina. Os recursos assistenciais são dimensionados segundo projeções da população potencialmente sob risco de doença cardiovascular e pulmonar, bem como a partir da demanda espontânea proveniente de serviços de emergência. Mantém 17 centros, entre laboratórios e grupos de pesquisa, e uma área de diagnóstico de alta complexidade reconhecida por seus equipamentos de última geração(7).

Anualmente o Hospital atende, em média, 260.000 consultas médicas; 37.000 atendimentos multiprofissionais; 13.000 internações; 5.000 cirurgias; dois milhões de exames de análises clínicas e 330.000 exames de diagnóstico de alta complexidade, o que o torna um contexto único para o atendimento de excelência de doenças do coração e do pulmão, desde a prevenção até o quadro clínico mais complexo ${ }^{(7)}$.

No que diz respeito ao atendimento $80 \%$ destina-se à pacientes provenientes do Sistema Único de Saúde (SUS) e $20 \%$ à pacientes de OPS e pacientes particulares. De acordo com o Diretor da Unidade de Faturamento (UFA) desse hospital o faturamento mensal corresponde a, aproximadamente, $50 \%$ de recursos provenientes do SUS e $50 \%$ das OPS e de pacientes particulares, a semelhança do que ocorre em outras instituições. Dados recentes indicaram que 91,7\% das contas hospitalares auditadas correspondeu à pacientes provenientes de OPS.

No Hospital, os médicos e enfermeiras auditores realizam a pré-análise conferindo todos os itens das contas relativas à pacientes de OPS e pacientes particulares, juntamente com os respectivos prontuários clínicos, e registram os dados auditados em planilhas eletrônicas. Cabe destacar que o prontuário clínico constitui-se em um dos principais instrumentos de trabalho para essa equipe.

Evidencia-se, inclusive, que parte significativa do 
pagamento de materiais, medicamentos e procedimentos é vinculada aos registros de enfermagem que devem ser consistentes, legíveis e objetivos para evitar glosas e perdas de faturamento ${ }^{(8)}$.

As glosas (correções) ocorrem quando qualquer situação gerar dúvidas em relação às regras e práticas adotadas pela instituição de saúde, observando-se conflito na relação entre convênio (OPS) e prestador de serviços (instituição hospitalar) ${ }^{(8)}$. Por sua vez, as perdas dizem respeito ao prejuízo financeiro decorrente da falta de cobrança de itens utilizados durante a internação do paciente, isto é, que são comprovados em documentos, tais como invólucros e notas fiscais, e registrados em prontuário, mas que não são devidamente lançados para efeito de faturamento ${ }^{(9)}$.

Ressalta-se que a prática de glosar itens do faturamento das contas constitui-se em perda significativa para o orçamento das instituições de saúde. Dessa forma, quando elas têm os valores dos serviços prestados glosados pelas OPS, podem munir-se de recursos de glosas, a fim de recuperar suas perdas econômicas $^{(8)}$.

Frente ao exposto, destaca-se que a auditoria de contas hospitalares é fundamental para a comprovação da realização dos procedimentos aos pacientes, pois fornece subsídios para viabilizar a cobrança junto às fontes pagadoras.

Considerando a relevância dessa temática, bem como a necessidade de divulgação e partilha de experiências de sucesso na administração pública em saúde, especialmente quando realizadas em uma unidade social de projeção e reconhecimento nacional e internacional, este estudo objetiva mapear e descrever o processo de formação de contas de um hospital público universitário, de nível terciário, especializado em cardiologia e pneumologia.

\section{MÉTODOS}

Trata-se de pesquisa exploratória, na modalidade de estudo de caso, realizada no Instituto do Coração (InCor) do Hospital das Clínicas da Faculdade de Medicina da Universidade de São Paulo (HCFMUSP).

Pesquisas desta natureza possibilitam a aproximação do pesquisador de um objeto ainda pouco conhecido para explorar os significados e contextos do fenômeno de investigação, buscando aprofundar o conhecimento em torno da realidade ${ }^{(10)}$. Tem por finalidade observar, descrever e documentar aspectos de uma situação ou realidade, bem como pesquisar fatores relacionados ao fenômeno em questão(11).

A escolha pelo estudo de caso, considerado de grande utilidade nas pesquisas exploratórias ${ }^{(12)}$, deve-se ao fato dele permitir investigar um fenômeno contemporâneo em profundidade e em seu contexto social, especialmente quando os limites entre o fenômeno e o contexto não são claramente evidentes ${ }^{(13)}$.

O estudo de caso constitui-se em um método abrangente, compreendendo lógica de planejamento desde a coleta, baseada em fontes de dados múltiplas e variadas (documentos, observação direta, entrevistas), análise e interpretação de dados até a apresentação do relatório final ${ }^{(13)}$.

Para sua consecução é preciso a permissão formal do responsável pela unidade social em estudo, pois o papel dos pesquisadores deve ser claro para aqueles que the prestam informações, não podendo ser confundido com elementos que inspecionam, avaliam ou supervisionam atividades $^{(14)}$.

Assim, após a anuência do Diretor da Unidade de Saúde Suplementar (USS) do InCor e aprovação do projeto pelo Comitê de Ética em Pesquisa da Escola de Enfermagem da Universidade de São Paulo, Certificado de Apresentação para Apreciação Ética - CAAE: 01737712.0.0000.5392 e parecer consubstanciado no 12980 , iniciou-se a coleta de dados.

O InCor foi inaugurado em 10 de janeiro de 1977 e, desde 1978, recebe o apoio da Fundação Zerbini, entidade sem fins lucrativos, que é responsável por captar, gerenciar e investir na sua estrutura, os recursos advindos da prestação de serviços do Instituto na assistência, ensino e pesquisa em cardiologia e pneumologia. A datar de sua inauguração tem prestado atendimento médico a pacientes de todos os Estados brasileiros, de toda América Latina, da América do Norte, Europa e Ásia, realizando mais de 38.350.287 exames de diagnóstico, 4.155.664 consultas ambulatoriais, 86.584 cirurgias, 255.202 estudos hemodinâmicos, incluindo cateterismos, angioplastias e ablação para tratamento de $\operatorname{arritmias}^{(5)}$.

Destaca-se que sua atuação na ciência e tecnologia, considerando as pesquisas clínicas e as básicas aplicadas, resultou em 5.720 estudos publicados em revistas nacionais e internacionais. Dessas pesquisas, mais de 20 originaram patentes ou produtos, como próteses, tubos, máquinas e equipamentos diversos atualmente em uso na área médica(5).

Segundo o Relatório de Gestão 2010 o Instituto realiza, anualmente, cerca de 280.000 consultas, 13.000 
internações, mais de 4.000 cirurgias e 2.800 .000 exames de diagnóstico. Conta com uma receita anual em torno de R\$369 milhões oriundas de recursos orçamentários e da prestação de serviços, no ramo da assistência está credenciado para atender a pacientes de, aproximadamente, 65 OPS $^{(6)}$.

Os pacientes do InCor recebem assistência médica e multiprofissional em programas de promoção, prevenção, diagnóstico, tratamento e reabilitação da saúde ${ }^{(5)}$. Para tanto, conforme anteriormente citado, possui 500 leitos destinados ao atendimento de pacientes com tratamento financiado por três fontes pagadoras: o SUS com 390 leitos (78\%), empresas de Saúde Suplementar (OPS) e pacientes particulares com 110 leitos (22\%).

Em 1999 instituiu a USS a fim de obter a adequada remuneração relativa a diárias, honorários profissionais, equipamentos, materiais, medicamentos, hemoterápicos e exames complementares destinados ao atendimento de pacientes provenientes de OPS e particulares por meio da auditoria de contas hospitalares e apresentação destas às fontes pagadoras.

A equipe da USS é composta por cinco auditores, dois médicos e três enfermeiras; dois analistas de contas médicas; uma digitadora; cinco auxiliares administrativos; uma supervisora de auditoria de contas médicas e recurso de glosas e dois analistas de glosas.

O Instituto dispõe de um sistema eletrônico, denominado Sistema Integrado de Informações (Si3), que fundamenta 0 processo de faturamento ao possibilitar a documentação do cuidado prestado ao paciente durante toda sua internação (Sistematização da Assistência de Enfermagem - SAE; anamnese, diagnóstico, evolução e prescrição médica; registros de outros profissionais da equipe de saúde; exames diagnósticos; procedimentos; materiais; medicamentos; gazes; equipamentos; diárias; honorários; taxas; entre outras). O Si3 gera relatórios que são impressos para compor o prontuário clínico do paciente.

Médicos e enfermeiras auditores registram, em planilhas eletrônicas que integram o banco de dados da USS, os dados obtidos a partir da análise das contas hospitalares prévias confrontadas com o prontuário clínico.

No período de maio a junho de 2012, a partir de momentos de observação não participante, procedeu-se o mapeamento das etapas integrantes do processo de formação de contas estudado. Finalizado o mapeamento as etapas foram descritas e validadas junto aos profissionais atuantes na UFA e na USS.

\section{RESULTADOS}

O processo de formação de uma conta hospitalar inicia-se na UFA assim que o paciente proveniente de OPS ou paciente particular é internado em umas das unidades do InCor.

Na UFA é aberto o prontuário contábil onde serão colocadas todas as notas de débito dos itens utilizados, guias e autorizações, bem como invólucros com as etiquetas contendo a descrição do material, número da nota fiscal, fornecedor, o fabricante, o código Agência Nacional de Vigilância Sanitária, lote e série dos materiais de alto custo utilizados durante a internação do paciente. Isto se faz necessário para comprovar que o Instituto não realiza o reprocessamento de materiais de alto custo.

Visando facilitar a conferência das contas hospitalares e minimizar o tempo para sua apresentação à fonte pagadora estabeleceu-se que para as internações de longa permanência seriam preparadas contas parciais a cada 10 dias, denominadas contas administrativas. Assim, como exemplo, se o paciente for hospitalizado por 15 dias terá duas contas, uma administrativa referente a 10 diárias e outra final relativa a cinco diárias.

No caso de pacientes particulares as contas são emitidas a cada quatro dias, e realizada a auditoria concorrente na unidade em que o mesmo está internado, pois a tabela de cobrança de taxas e serviços é extensa. Esta medida facilita a pré-análise e a apresentação das contas aos pacientes que no momento da alta hospitalar terão conhecimento da quantia a ser paga.

Há outra forma de cobrança desenvolvida na UFA, que é regida por contratos firmados entre o InCor e as OPS, denominada pacote. Nos pacotes não constam o detalhamento dos itens a serem cobrados uma vez que é estabelecido um preço fechado para a realização de um dado procedimento, com prazo determinado de internação. Entretanto, caso ocorram complicações clínicas decorrentes do procedimento, demandando o prolongamento da internação do paciente, torna-se necessária a abertura das contas parciais e finais.

Em síntese, a UFA emite uma conta prévia à USS a cada 10 dias (no caso de pacientes provenientes de OPS), quatro dias (pacientes particulares) ou quando o paciente receber alta antes de completar esses períodos, anexando junto ao prontuário contábil, o seu prontuário clínico para que se realize a pré-análise.

No InCor, o fluxo mensal corresponde, em média, a 
300 contas divididas em três subgrupos: clínico, cirúrgico e de procedimentos diagnósticos e/ou terapêuticos realizados no Serviço de Hemodinâmica. As contas de pacientes que inicialmente realizaram procedimentos nesse Serviço e posteriormente foram encaminhados à cirurgia, são classificadas como do subgrupo cirúrgico.

Os auditores conferem todos os itens das contas prévias juntamente com o prontuário clínico do paciente e registram os dados em planilhas eletrônicas distintas que possuem itens comuns e específicos. Para favorecer a visualização comparativa dos componentes destas planilhas apresenta-se o Quadro 1.

Após a equipe de auditoria realizar a pré-análise, as contas são devolvidas à UFA com a indicação dos itens que necessitam de correção. Realizadas as correções a UFA encaminha as contas à USS para apresentação destas as OPS para a condução da auditoria externa (médica e de enfermagem).

Ao final da negociação entre a USS e as OPS são identificadas as glosas e as contas enviadas à UFA para os ajustes finais, permanecendo apenas os itens acordados entre os auditores internos e externos para emissão das notas fiscais e cobrança. Na Figura 1 esquematiza-se o processo de formação de conta hospitalar descrito.

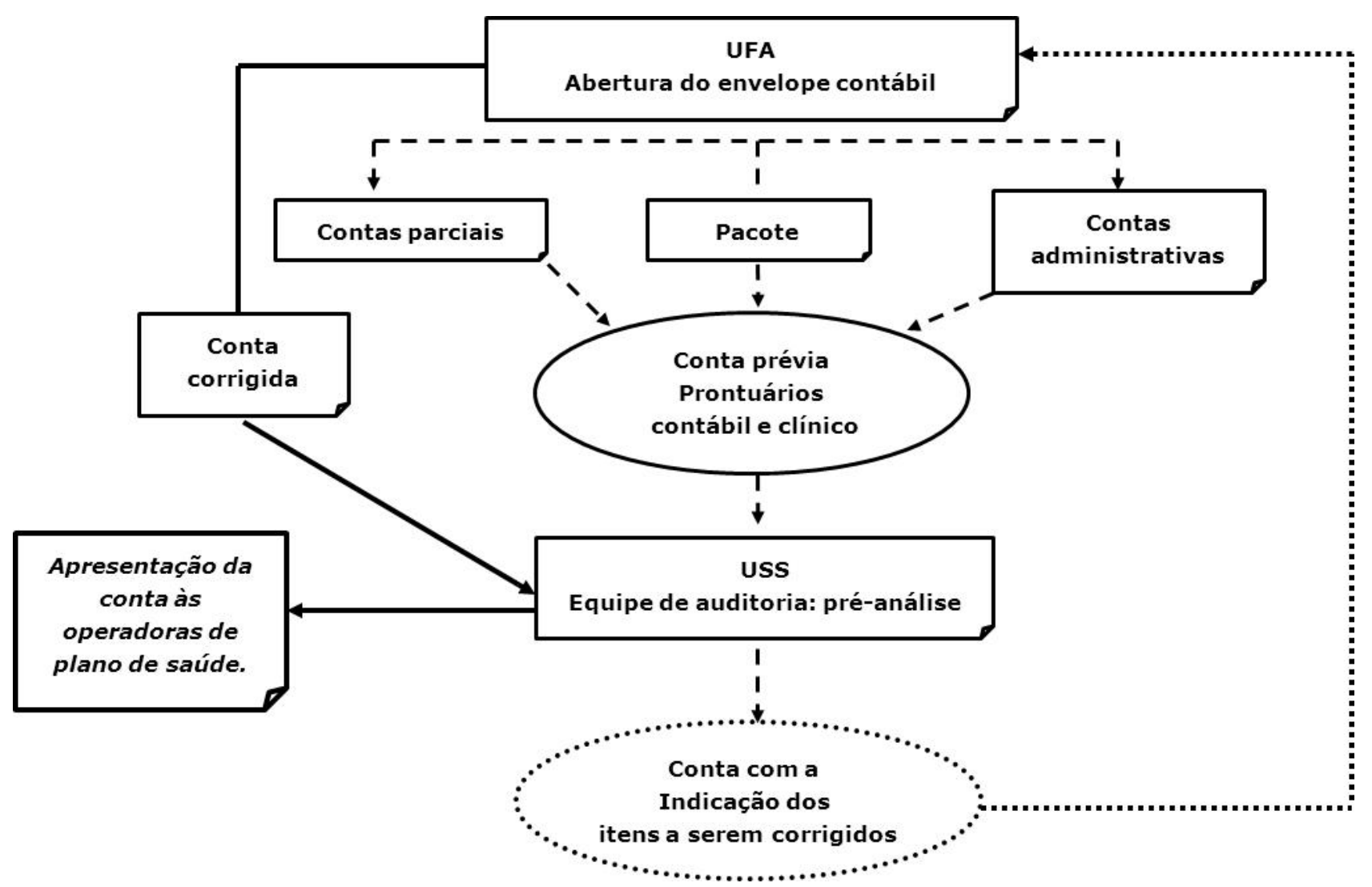

Figura 1: Síntese do processo de formação da conta hospitalar do Incor do HC-FMUSP. São Paulo, SP, Brasil, 2012.

Por fim, tendo como referência o ano de 2011, avaliou-se o tempo de permanência das contas hospitalares, durante o processo de formação, na UFA e USS, conforme demonstrado na Tabela 1.

Tabela 1: Distribuição do tempo de permanência, em dias, da conta hospitalar na UFA e USS do InCor HCFMUSP até a sua apresentação às fontes pagadoras. São Paulo, SP, Brasil, 2012.

\begin{tabular}{cccccc}
\hline Variáveis & Média & DP & IC 95\% & Mediana & Min-Máx \\
\hline UFA & 23,08 & 27,94 & 22,$0 ; 24,2$ & 16 & $0-703$ \\
USS & 6,96 & 8,58 & 6,$6 ; 7,3$ & 5 & $0-166$ \\
UFA + USS & 30,03 & 30,63 & 28,$9 ; 31,2$ & 22 & $1-707$ \\
Tempo total (em dias) & 33,92 & 31,72 & 32,$7 ; 35,1$ & 26 & $0-713$ \\
\hline
\end{tabular}


Quadro 1: Itens registrados pela equipe de auditores, médicos e enfermeiras, contidos nas planilhas eletrônicas que integram o banco de dados da USS do InCor. São Paulo, SP, Brasil, 2012.

\begin{tabular}{|c|c|c|}
\hline Itens registrados por médicos & Itens registrados por enfermeiras & $\begin{array}{l}\text { Itens comuns } \\
\text { à equipe de } \\
\text { auditoria }\end{array}$ \\
\hline Sexo & $\begin{array}{l}\text { Serviço de enfermagem (pré e pós-análise, ajustes e glosas): cuidados de } \\
\text { enfermagem realizados aos pacientes durante o período de internação } \\
\text { (punções venosas; administração de medicamentos subcutâneos e/ou } \\
\text { endovenosos; controle de diurese; curativos; enteroclisma; administração de } \\
\text { dieta por via enteral; realização de orientações de enfermagem; cateterismo } \\
\text { vesical; tricotomia; retirada de pontos, drenos, fios e cateteres; instalação de } \\
\text { hemodiálise, inalação, controle de débito cardíaco, entre outras). }\end{array}$ & $\begin{array}{l}\text { Registro } \\
\text { Hospitalar }\end{array}$ \\
\hline Idade & $\begin{array}{l}\text { Equipamentos (pré e pós-análise, ajustes, divergências e glosas): inclusão de } \\
\text { taxa referente à utilização de equipamentos, por dia ou hora, como exemplos, } \\
\text { bomba de infusão, respirador, transdutor, polígrafo, telemetria, monitorização } \\
\text { de pressão não invasiva marcapasso temporário, balão intra-aórtico, oxímetro. }\end{array}$ & $\begin{array}{l}\text { Nome do } \\
\text { paciente }\end{array}$ \\
\hline Óbito & $\begin{array}{c}\text { Gases (pré e pós-análise, ajustes e glosas): oxigênio, ar comprimido e oxido } \\
\text { nítrico de acordo com o tempo de utilização. }\end{array}$ & Nome da OPS \\
\hline Diagnóstico médico & $\begin{array}{l}\text { Materiais utilizados na internação (pré e pós-análise, ajustes, divergências e } \\
\text { glosas), tais como, agulhas, seringas, luvas, equipos, torneirinhas, gaze. }\end{array}$ & $\begin{array}{l}\text { Data de início } \\
\text { da conta }\end{array}$ \\
\hline $\begin{array}{l}\text { Diárias (apartamento, UTI, total), taxas administrativas, exames (como } \\
\text { exemplos: patologia clínica, diagnóstico por imagem e métodos gráficos), } \\
\text { hemoterapia, honorários médicos, fisioterapia (motora e respiratória), } \\
\text { verificação das autorizações das OPS para a realização de procedimentos, dos } \\
\text { códigos de procedimentos que devem ser preenchidos conforme as tabelas } \\
\text { previamente acordadas e utilização de materiais de alto custo. }\end{array}$ & $\begin{array}{l}\text { Medicamentos utilizados na internação (pré e pós-análise, ajustes e glosas): } \\
\text { fármacos vasoativos, antibióticos, soluções para diluição, eletrólitos. }\end{array}$ & $\begin{array}{l}\text { Data de fim da } \\
\text { conta }\end{array}$ \\
\hline Valor final total da conta & $\begin{array}{c}\text { Materiais utilizados no Centro Cirúrgico: pré e pós-análise, ajustes, } \\
\text { divergências e glosas. }\end{array}$ & $\begin{array}{l}\text { Valor inicial da } \\
\text { conta enviada } \\
\text { pela UFA }\end{array}$ \\
\hline Glosa final total & $\begin{array}{c}\text { Medicamentos utilizados no Centro Cirúrgico: pré e pós-análise, ajustes e } \\
\text { glosas. }\end{array}$ & \multirow{8}{*}{$\begin{array}{l}\text { Valor da conta } \\
\text { ajustado }\end{array}$} \\
\hline Grupo cardíaco pertencente & $\begin{array}{l}\text { Materiais utilizados no } \\
\text { Serviço de Hemodinâmica: pré e pós-análise, ajustes, divergências e glosas. }\end{array}$ & \\
\hline Data de chegada da conta à USS & \multirow{6}{*}{$\begin{array}{c}\text { Medicamentos utilizados no Serviço de Hemodinâmica: pré e pós-análise, } \\
\text { ajustes e glosas. }\end{array}$} & \\
\hline $\begin{array}{l}\text { Tipo de conta } \\
\end{array}$ & & \\
\hline Cobrança: administrativa (10 e quatro dias) e final & & \\
\hline Período da alta do paciente até a emissão da conta à UFA & & \\
\hline Tempo em que a conta permanece na USS para auditoria & & \\
\hline Tempo total considerando a auditoria e emissão da conta pela UFA & & \\
\hline Data de fechamento da conta pela UFA & & $\begin{array}{c}\text { Tipo de } \\
\text { internação }\end{array}$ \\
\hline
\end{tabular}

Rev. Eletr. Enf. [Internet]. 2014 jul/set;16(3):558-65. Disponível em: http://dx.doi.org/10.5216/ree.v16i3.23487. - doi: 10.5216/ree.v16i3.23487. 
O tempo total de formação das 2.613 contas hospitalares variou de zero a 713 dias, com média de 33,92 (DP $\pm 31,72$ ) e mediana de 26 dias. Percebe-se que o maior tempo de permanência ocorreu na UFA com variação de zero a 703, média de 23,08 (DP士 27,94) e mediana de 16 dias.

\section{DISCUSSÃO}

O Incor do HC-FMUSP, a exemplo do que ocorre em outros hospitais, públicos e privados, que prestam serviços às OPS, investe na auditoria de contas visando à adequada remuneração do atendimento realizado. No momento da pré-análise das contas a equipe de médicos e enfermeiras auditores realiza correções para fundamentar a cobrança dos procedimentos e evitar glosas e perdas de faturamento, que comprometeriam a saúde econômica da Instituição.

Diante da globalização e do cenário político financeiro brasileiro, o setor de saúde tem procurado novas alternativas para a gestão, focalizando a necessidade das organizações adaptarem-se a um mercado cada vez mais competitivo ${ }^{(15)}$.

Os estabelecimentos prestadores de serviços de saúde constituem-se em empresas complexas que necessitam adaptar-se a esse novo cenário e tornar-se flexíveis para incorporarem estratégias capazes de atender ao usuário interno e externo ${ }^{(16)}$.

Compreende-se que a exigência por parte dessas instituições em relação à atuação dos seus profissionais de saúde na área contábil e financeira, tem o propósito de adequar os custos e incrementar recursos mediante o enfoque empresarial e mercadológico(17).

Assim, em uma de suas perspectivas, a auditoria pode ser utilizada como uma ferramenta de controle e regulação da utilização de serviços de saúde focalizando, especialmente na área privada, o controle dos custos da assistência prestada(18)

Nessa ótica, a auditoria propicia além da detecção dos problemas apresentados nos prontuários $e$ possibilita, por meio dos relatórios de avaliação, a orientação para a equipe e a instituição de saúde, quanto ao registro apropriado das ações profissionais e o respaldo ético e legal, frente aos conselhos, as associações de classe e a justiça ${ }^{(19)}$.

Destarte a auditoria extrapolaria o controle administrativo e financeiro das organizações e tornar-seia também, uma atividade educativa, pois os profissionais responsáveis por sua realização promoveriam ações enfatizando as causas que levaram ao erro ao invés de buscar apenas o responsável pela falha $^{(20)}$.

Ao realizar auditoria interna a enfermeira pode detectar falhas e para que estas não se tornem recorrentes é fundamental o estabelecimento de parcerias com o Serviço de Educação Continuada determinando um plano de ação compreendendo reeducação, treinamentos, conscientização, valorização dos pontos positivos, reflexão sobre os negativos e orientação contínua de toda a equipe ${ }^{(21)}$.

Atividades destinadas à educação permanente representam uma boa estratégia, especialmente se conduzirem os trabalhadores de enfermagem a uma reflexão sobre sua prática, bem como em relação as possibilidades presentes no cotidiano do trabalho para consecução de uma assistência mais qualificada e pautada na ética ${ }^{(3)}$.

Na unidade social estudada, visto que somente as enfermeiras documentam os dados pré e pós-análise, ajustes e glosas, e os médicos registram apenas a glosa total, seria de fundamental importância que a equipe de auditoria da USS harmonizasse os conteúdos documentados nas respectivas planilhas eletrônicas e realizasse a documentação detalhada dos itens glosados pelas OPS. Isso propiciaria o desenvolvimento de atividades educativas na Instituição visando o registro adequado das atividades desenvolvidas, por toda equipe de saúde, no cuidado ao paciente e o estabelecimento conjunto de estratégias para evitar o comprometimento do faturamento.

Evidentemente não se pode eximir os profissionais de saúde da responsabilidade ética, legal, jurídica e comunicativa da documentação das ações prestadas ou das justificativas por elas não terem sido realizadas. Todavia, investimentos compartilhados para a realização de atividades educativas visando à melhoria dos registros contribuirão, sobremaneira, para minimizar as divergências ao se comparar o prontuário clínico e a conta do paciente ${ }^{(9)}$.

Considerados o quadro de profissionais atuantes na USS e os 110 leitos destinados aos pacientes de OPS constata-se a pertinência de ações Institucionais visando à realização da auditoria concorrente das contas desses pacientes, do mesmo modo como são feitas com as contas dos pacientes particulares.

Propõe-se, também, que seja averiguada a alternativa do auxiliar administrativo da unidade onde o paciente se encontra internado realizar o lançamento das notas de débito ao invés desta ação ser realizada na 
USS, o que minimizaria o tempo de formação das contas.

Acredita-se que tais medidas facilitariam aos auditores a pré-análise das contas hospitalares e favoreceria a realização de atividades educativas sistemáticas junto à equipe responsável pelos registros no prontuário clínico, principal fonte de comprovação para o recebimento do pagamento dos serviços prestados.

\section{CONCLUSÃO}

A condução deste estudo permitiu mapear e descrever, detalhadamente, o processo de formação de contas hospitalares do Incor do HC-FMUSP, unidade

\section{REFERÊNCIAS}

1. Camelo SHH, Pinheiro A, Campos D, Oliveira TL, Auditoria de enfermagem e qualidade da assistência à saúde: uma revisão de literatura. Rev. Eletr. Enf. [Internet]. 2009 [acesso em 21 jan 2013]; 11(4) 1018-25. Disponível em:

http://www.fen.ufg.br/revista/v11/n4/v11n4a28.htm. 2. Scarparo AF, Ferraz CA. Auditoria de enfermagem: identificando sua concepção e métodos. Rev. Bras. Enferm. [Internet]. 2008 [acesso em: 13 fev 2013]; 61(3): 302-5. Disponível em: http://dx.doi.org/10.1590/S003471672008000300004 .

3. Dias TCL, Santos JLG, Cordenuzzi OCP, Prochnow AG. Auditoria em enfermagem: revisão sistemática da literatura. Rev. Bras. Enferm. [Internet]. 2011 [acesso em: 11 Abr 2014] ; 64(5): 931-7. Disponível em: http://dx.doi.org/10.1590/S003471672011000500020.

4. Scarparo AF, Ferraz CA, Chaves LDP. Auditoria como ferramenta de Avaliação dos Serviços de enfermagem. In: Eucléa Gomes Vale; Organizadoras: Lima JR, Felli VEA. (Org.). Programa de Atualização em Enfermagem (PROENF): Gestão. $1^{a}$ ed. Porto Alegre: Artmed/Panamericana editora LTDA, 2011;1(1): 91-121. 5. Motta ALC. Auditoria de Enfermagem. In: Auditoria enfermagem nos hospitais e seguradoras de saúde $5^{a}$. ed. São Paulo: Iátria, 2010. Cap 01 p.17-9.

6. Instituto do Coração (InCor) do Hospital das Clínicas da Faculdade de Medicina da Universidade de São Paulo (HCFMUSP) [Internet]. São Paulo: InCor-HCFMUSP [acesso em: $10 \mathrm{fev}$ 2013]. Conheça quem somos. Disponível em:

http://www.incor.usp.br/sites/incor2013/index.php/conhecaquem-somos

7. Instituto do Coração (InCor) do Hospital das Clínicas da Faculdade de Medicina da Universidade de São Paulo (HCFMUSP) [Internet]. São Paulo: InCor-HCFMUSP [acesso em: $10 \mathrm{fev}$ 2013]. Relatório de Gestão Instituto do Coração 2010. Disponível em:

http://www.incor.usp.br/sites/incor2013/docs/relatorios_pngs/Re latorio-PNGS-2010.pdf

8. Rodrigues VA, Perroca MG, Jericó MC. Glosas hospitalares: importância das anotações de Enfermagem. Arq. Ciênc. Saúde. 2004;11(4):210-4.

9. Guerrer GFF. Auditoria de contas em um hospital de ensino especializado em cardiologia e pneumologia: um estudo de caso. [dissertação]. São Paulo (SP): Escola de Enfermagem/USP; 2012. 82 p.

10. Trivinos ANS. Introdução à pesquisa em Ciências Sociais. São Paulo: Atlas; 2008. Alguns temas no desenvolvimento de pesquisa. p. 91-115.

11. Polit DF, Beck CT. Fundamentos de pesquisa em Enfermagem. Avaliação de evidências para a prática da enfermagem. 7a ed. Porto Alegre: Artmed; 2011.

12. Ventura MM. O Estudo de Caso como Modalidade de social de projeção e relevância nacional e internacional. Propiciou a realização de propostas de mudanças exequíveis evidenciando possibilidades de revisão e melhoria visando minimizar o tempo de apresentação de contas às fontes pagadoras.

Ao conferir visibilidade à dinâmica deste processo, fundamental para o equilíbrio econômico-financeiro do Instituto, torna-se esse conhecimento de domínio público e acessível a outras organizações de saúde que queiram incrementar o seu faturamento e reduzir as divergências entre o prontuário clínico e a conta hospitalar do paciente.

Pesquisa. Rev SOCERJ. [Internet]. 2007 [acesso em 15 jan 2013]; 20(5):383-6. Disponível em: http://sociedades.cardiol.br/socerj/revista/2007_05/a2007_v20_ n05_art10.pdf.

13. Yin RK. Estudo de caso: Planejamento e Método. $4^{\mathrm{a}}$ ed. Porto Alegre: Bookman, 2010.

14. Martins GA. Estudo de caso: uma reflexão sobre a aplicabilidade em pesquisas no Brasil. RCO - Revista de Contabilidade e Organizações - FEARP/USP 2008; 2(2); 8-18. 15. Paim CRP, Ciconeli RM, Auditoria da avaliação da qualidade dos serviços de saúde. Revista de Administração em Saúde. 2007; 9(36): 85-91.

16. Tronchin DMR, Melleiro MM, Takahashi RT. A qualidade e a avaliação dos serviços de saúde e de enfermagem. In: Kurcgant P. Gerenciamento em enfermagem. 2a ed. Rio de Janeiro: Guanabara Koogan, 2010. Cap. 7 p 71-84.

17. Scarparo AF, Ferraz CA, Chaves LDP, Gabriel CS. Tendências da função do enfermeiro auditor no mercado em saúde. Texto Contexto Enferm. [Internet] 2010[acesso em: 02 fev 2013]; 19(1): 85-92. Disponível em: http://dx.doi.org/10.1590/S010407072010000100010 .

18. Pinto KA, Melo CMM de. A prática da enfermeira auditora em saúde. Rev Esc Enferm USP. [Internet]. 2010 [acesso em: $10 \mathrm{fev}$ 2013]; 44(3): 671-8. Disponível em:

http://dx.doi.org/10.1590/S0080-62342010000300017. 19. Setz VG, D'Innocenzo M. Avaliação da qualidade dos registros de enfermagem no prontuário por meio da auditoria. Acta Paul. Enferm. [Internet]. 2009 [citado 08 Abr 2014]; 22(3): 313-7. Disponível em: http://dx.doi.org/10.1590/S010321002009000300012.

20. Buzatti CV, Chianca TC. Auditoria em enfermagem: erros e custos envolvidos nas anotações. Rev. Nursing 2005; 90(8); 4650.

21. Silva PC, Casa ECGS. Auditoria Interna em enfermagem e educação continuada: um feedback positivo. Rev Enferm UNISA. 2006; (7); 48-51.

Artigo recebido em 03/04/2013. Aprovado para publicação em 10/04/2014. Artigo publicado em 30/09/2014. 